\title{
X-ray properties in massive galaxy clusters: XMM-Newton observations of the REFLEX-DXL sample (Corrigendum)
}

\author{
Y.-Y. Zhang ${ }^{1}$, H. Böhringer ${ }^{1}$, A. Finoguenov ${ }^{1}$, Y. Ikebe ${ }^{1,2}$, K. Matsushita ${ }^{1,3}$, P. Schuecker ${ }^{1}$, \\ L. Guzzo ${ }^{4}$, and C. A. Collins ${ }^{5}$ \\ 1 Max-Planck-Institut für extraterrestrische Physik, Giessenbachstraße, 85748 Garching, Germany \\ e-mail: yyzhang@astro.uni-bonn.de \\ 2 National Museum of Emerging Science and Innovation, Tokyo, Japan \\ 3 Tokyo University of Science, Tokyo, Japan \\ 4 INAF - Osservatorio Astronomico di Brera, Merate/Milano, Italy \\ 5 Liverpool John Moores University, Liverpool, UK
}

A\&A 456, 55-74 (2006), DOI:10.1051/0004-6361:20053650

ABSTRACT

The article by Zhang et al. (2006, A\&A, 456, 55) contains an error in Fig. 5 on page 64, in which we inadvertently re-called the mass distribution of RXCJ0528.9-3972. We note that this does not affect any results in Zhang et al. (2006). Nevertheless, we regret this error and provide an update of Fig. 5 here.

Key words. cosmology: observations - galaxies: clusters: general - X-rays: galaxies: clusters - dark matter - errata, addenda

\section{References}

Zhang, Y.-Y., Böhringer, H., Finoguenov, A., et al. 2006, A\&A, 456, 55 
A\&A 527, C2 (2011)
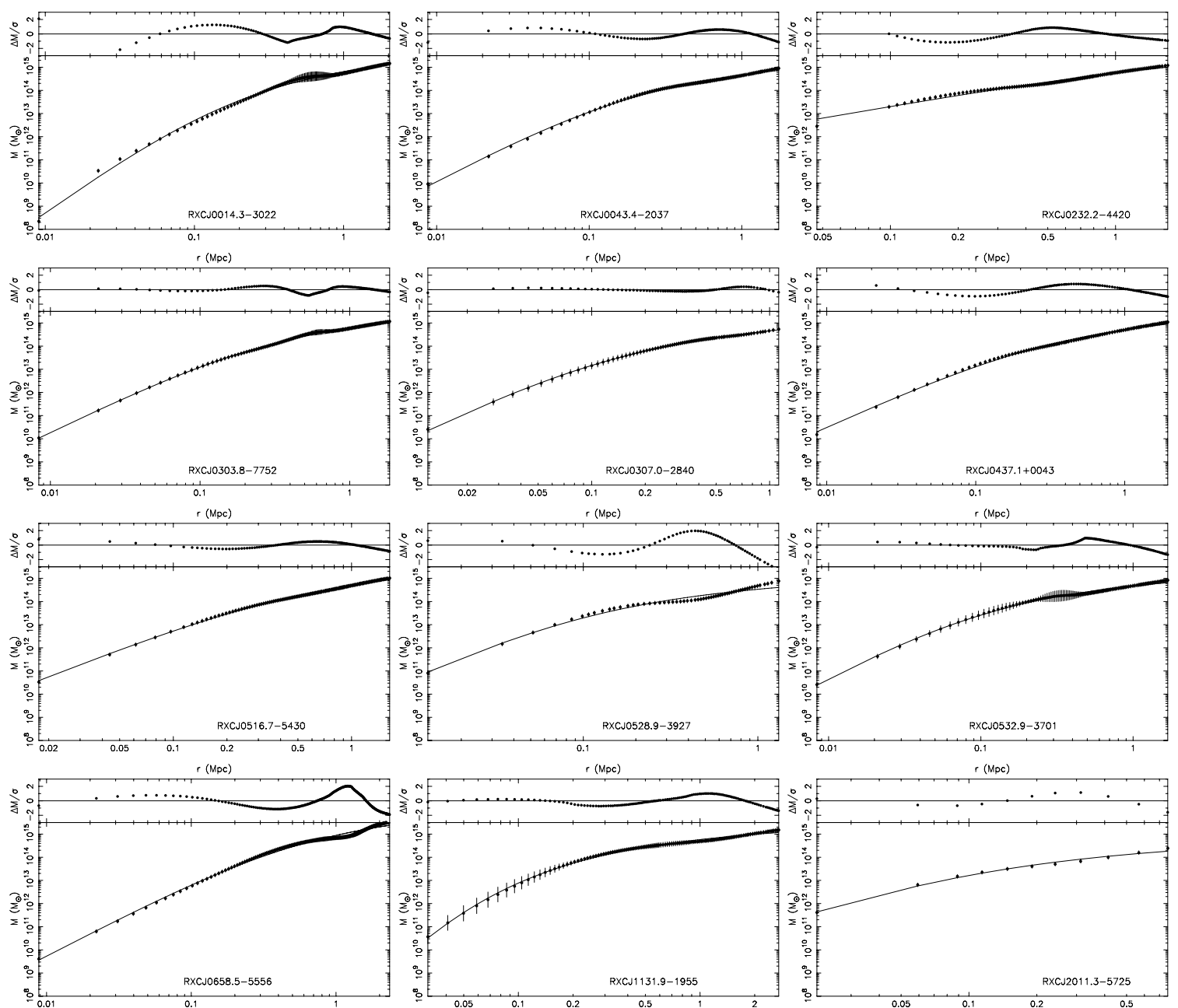

(

$r(\mathrm{Mpos})$
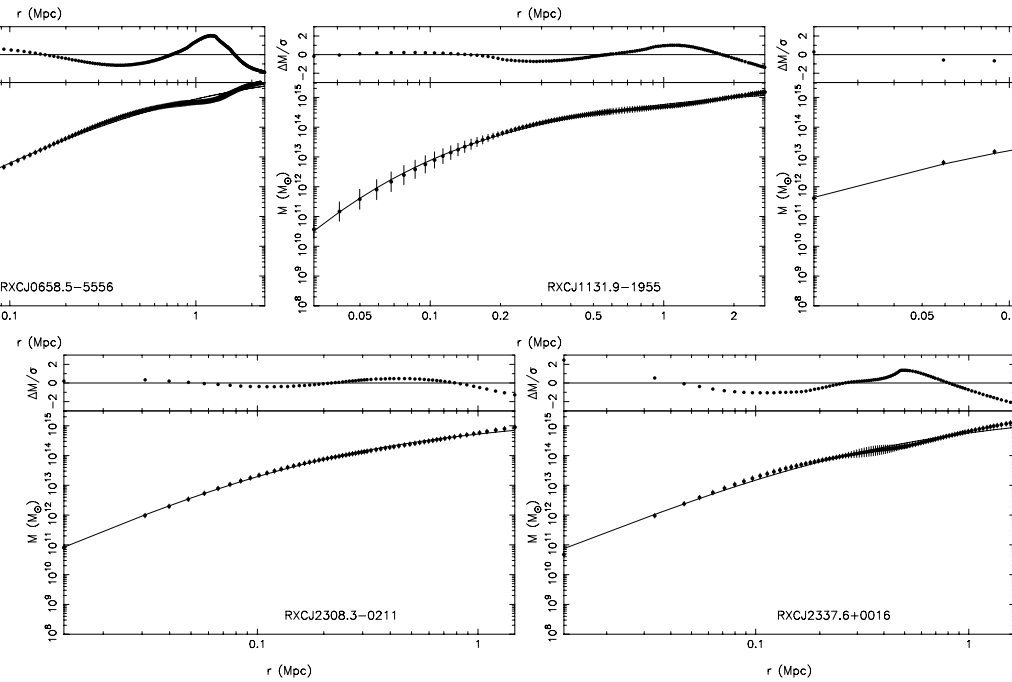

RXCJ2011.3-5725

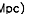

Fig. 5. Measured mass profiles of the REFLEX-DXL clusters and their best fits by the extended-NFW models. Residuals scaled by the data uncertainties are plotted in the upper part of the diagrams. 